\title{
Design and Flow Simulation of a New Micromixer*
}

\author{
Chen-I HUNG ${ }^{* *}$, Ke-Chin WANG ${ }^{* * *}$ and Chin-Kwun $\mathrm{CHYOU}^{* * *}$
}

\begin{abstract}
The complete mixing of two or more different fluids in the microscopic scale within a reasonable time period plays an important role in the Micro-Total-Analysis-System ( $\mu$ TAS). The objective of this study is to propose a new design of a passive micromixer by using flowfield simulations through Computational Fluid Dynamics (CFD) techniques. The primary idea of designing the micromixer is to add feedback side-channels on both sides of the main channel. Through the feedback side-channels, the fluids are guided to flow back into the main channel and to push the main stream and, therefore, the mixing effect will be produced and enhanced. By simulating the flow field in the micromixer, the important design parameters and the operational conditions, such as the channel geometry, the position of side-channel, and the inlet velocity, can be examined.
\end{abstract}

Key Words: Micromixer, $\mu$ TAS, Feedback Side Channel, CFD

\section{Introduction}

According to the operational functions, biochips can be classified into the following categories, such as gene chip, Lab-on-a-chip, and protein chip. Among them, Labon-a-chip is an application of miniaturized total chemical analysis system (i.e., $\mu$ TAS) and was first proposed by Manz et al. ${ }^{(1)}$ The concept of $\mu$ TAS is to integrate some instruments needed in traditional biochemical analysis, such as micro-pump, micro-actuator, micro-valve, microsensor and micromixer which are fabricated by using the Micro-Electro-Mechanical-Systems (MEMS) techniques, and then the pretreatment, transmission, mixing, reaction, separation, and detection of the sample can be proceeded on a single chip. The advantages of using the Lab-ona-chip on biochemical analysis are strong system stability, reduced reagent amount, and the saving of manpower and time. Due to the wide applications of fluid transmission, the development of micro-fluidic devices and systems arising day by day, and micromixers are the most important components in the biomedical and chemical investigations. Therefore, efficient and effective mixing of two or more different fluids in a micro-scale region has become an interesting challenge of the development of biomedical investigations.

* Received 22nd January, 2004 (No. 04-5015)

** Department of Mechanical Engineering, National ChengKung University, Tainan 701, Taiwan. E-mail: cihung@mail.ncku.edu.tw

*** Graduate student, Department of Mechanical Engineering, National Cheng-Kung University, Tainan 701, Taiwan
In the macro-scale flow field the contact area between two different fluids can be increased by producing the flow turbulence, therefore the mixing effect can be enhanced. However, in the micro-scale flow field, the flow velocity and length scales are smaller than the corresponding ones in the macro-scale field, and the corresponding Reynolds number in micro-scale systems is smaller than the critical Reynolds number at which the turbulence will occur. Therefore, the possibility to improve the mixing effects in the micro-scale field by producing the flow turbulence is excluded. So, in laminar flow regime, how to efficiently and effectively mix two or more different fluids would be a major challenge of designing a micromixer.

The current development of micromixers could be classified into two categories by the mixing methods adopted: active mixers ${ }^{(2),(3)}$ and passive mixers ${ }^{(4)-(7)}$. The mixing effects of active mixers are produced by the input of external energy. Therefore, the mixing efficiency can be adjusted by changing the input energy. Yang et al. ${ }^{(2)}$ first reported a micromixer using turbulence principle and liquids were mixed in a chamber with oscillating diaphragm driven by a high-frequency PZT. Yang et al. ${ }^{(3)}$ designed and fabricated an active micromixer in which mixing occurred directly from ultrasonic vibration. The passive mixer produces mixing effect by simply modifying the mixer geometry or changing fluid properties, without external energy input. Miyake et al. ${ }^{(4)}$ arranged 400 micronozzles on the channel bottom and a reagent is injected through these nozzles that can make many micro-plumes, and hence, the contact surface between liquids is drastically increased. Knight et al. ${ }^{(5)}$ used nanoscale submerged 
fluid jets to enhance mixing. Schwesinger et al. ${ }^{(6)}$ developed an integrated system, which consists of different modular microcomponents that allows a homogeneous mixing of different fluids. Veenstra et al. ${ }^{(7)}$ designed a new micromixer which is suitable for mixing slowly diffusing compounds in systems with low flow rates. In this study, a design of the geometry of a new passive-type micromixer is proposed. Flow fields in the proposed micromixer are simulated using the Computational Fluid Dynamics (CFD) techniques. From the numerical simulations, the geometry design parameters and the inlet flow velocity of micromixer for the efficient mixing could be determined.

\section{Mathematical Analysis}

To improve the mixing effect of a micromixer, the present study proposes a new design of micromixer by adding feedback side channels on both sides of the main flow channel of the micromixer. The schematic diagrams of the proposed micromixer and convergent nozzle used for the main channel and feedback side channels are depicted in Fig. 1 (a) and (b) with related dimensions indicated. Through the feedback side-channels, some fluid will be guided to flow back into the main channel, so as to produce an impact on the main stream. Then the main stream will be bended and an apparent vortex will be formed, and therefore, the mixing effect will be strongly

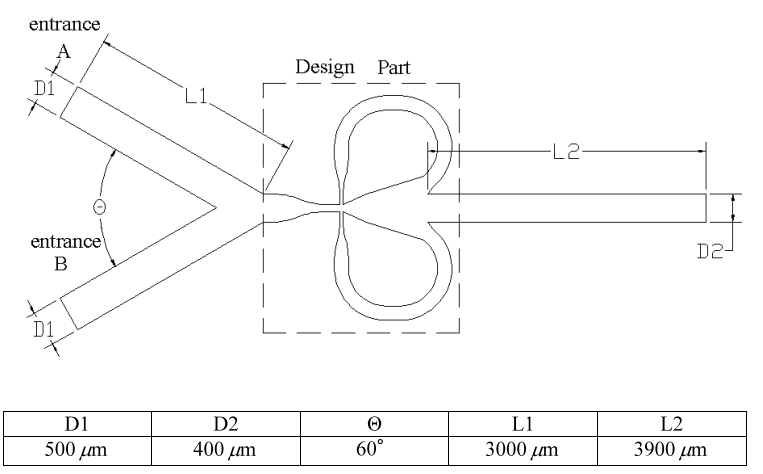

(a)

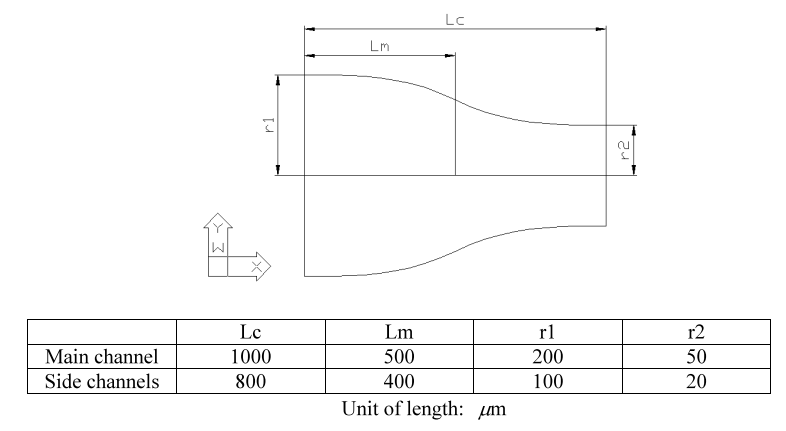

(b)

Fig. 1 Schematic representation of (a) the new type micromixer with the addition a feedback side-channel loop and (b) a convergent nozzle enhanced.

\section{1 Governing equations}

In this study, the flow in a micromixer is assumed to be steady, two-dimensional, laminar and incompressible. The temperature field is assumed as an isothermal distribution. The properties of fluid, such as viscosity and mass diffusivity, are assumed to be constant. The body force due to gravity is neglected. There is no chemical reaction between two different fluids and only the concentration gradient between two fluids is considered. The two inlet streams are assumed as the same fluids, but with different concentrations. That is, fluid $A$ flows into the left inlet channel with maximum molar fraction of material $A$, $C_{A \text {,max }}$, and in the right inlet channel, fluid $B$ flows with minimum molar fraction of material $A$, i.e., $C_{A, \min }(=0)$. The inlet velocity $\left(V_{0}\right)$ and the width of the main channel $\left(D_{2}\right)$ are chosen as the characteristic velocity and characteristic length, respectively. The dimensionless quantities adopted in this study are listed as follows:

$$
\begin{aligned}
\vec{V}^{*} & =\frac{\vec{V}}{V_{0}}, \quad p^{*}=\frac{p-p_{0}}{\rho V_{0}^{2}}, \quad C_{A}^{*}=\frac{C_{A}-C_{A, \min }}{C_{A, \max }-C_{A, \min }}, \\
\nabla^{*} & =D_{2} \nabla ; \\
\operatorname{Re} & =\frac{\rho V_{0} D_{2}}{\mu}, \quad S c=\frac{\mu}{\rho D_{A}} .
\end{aligned}
$$

In the above expressions, $\vec{V}$ is the velocity vector, $\nabla$ the gradient vector, $p$ the pressure, $p_{0}$ the referenced pressure, $C_{A}$ the molar concentration of material $A, \mu$ the fluid viscosity, $\rho$ the fluid density, $D_{A}$ the mass diffusivity of material $A$. The superscript "*" represents the dimensionless quantities. $R e$ and $S c$ are Reynolds number and Schmidt number, respectively.

Based on the above-mentioned assumptions, the dimensionless governing equations for mass, momentum, and concentration conservation may be expressed as follows:

Continuity equation:

$$
\nabla^{*} \cdot \vec{V}^{*}=0
$$

Momentum equation:

$$
\vec{V}^{*} \cdot \nabla^{*} \vec{V}^{*}=-\nabla^{*} p^{*}+\frac{1}{R e} \nabla^{* 2} \vec{V}^{*}
$$

Diffusion equation

$$
\vec{V}^{*} \cdot \nabla^{*} C_{A}^{*}=\frac{1}{\operatorname{ReSc}} \nabla^{* 2} C_{A}^{*}
$$

\subsection{Boundary conditions}

For all cases studied, a uniform velocity distribution is specified at the two inlets. The maximum $\left(C_{A}^{*}=1\right)$ and minimum $\left(C_{A}^{*}=0\right)$ concentration is specified at the left and right inlet boundary, respectively. At the outlet of main channel, the reference pressure is specified, and in this study, it is taken as zero gauge pressure, i.e., $p_{e}=p_{0}=1 \mathrm{~atm}=1 \times 10^{5} \mathrm{~Pa}$. For the outlet concentration, a non-varying condition is specified. On wall boundaries, 
the non-penetrating and no-slip conditions should be satisfied.

\section{Numerical Method}

In numerical simulations, the commercial code CFD$\mathrm{ACE}(\mathrm{U})^{(8)}$ has been chosen to calculate the flow fields in the proposed new-type micromixer. $\mathrm{CFD}-\mathrm{ACE}(\mathrm{U})$ employs a structured, multi-domain, body-fitted-coordinatedsystem approach to simulate flows in complex geometric configurations. The aspects of CFD-ACE(U) structured flow solver solution methodology are briefly described as follows: (1) Cell-centered control-volume approach to discretize the governing equations, (2) Pressure-based solution scheme, (3) Iterative nature of the solution scheme.

The grid system inside the computational domain is generated by the structured grid generation method. The complicated geometry of the new type micromixer is curve fitted by using the non-uniform rational B-splines (NURBS). The grid systems generated inside the computational domain are structured. Systems of total numbers of grids of 13411, 30127,53681 , and 64940 are used for testing the grid independency. Computational results of mole fraction distribution, the mixing efficiency, and the pressure drop at the exit show little difference between the total grid system of 53681 and $64940^{(9)}$. Therefore, the system of 53681 grids is used for all numerical computations in the study.

\section{Definition of Mixing Efficiency}

To quantitatively evaluate the performance of a micromixer with different design parameters, a mixing efficiency should be defined to judge the mixing effect in a micromixer. From the computational results of concentration fields, the mixing efficiency is defined as

$$
\varepsilon_{\text {mixing }}=1-\frac{1}{L} \int_{0}^{L}\left|\frac{C_{A, x}^{*}-0.5}{C_{A, \max }^{*}-0.5}\right| d x
$$

where $\varepsilon_{\text {mixing }}$ is the mixing efficiency, $C_{A \text {, max }}^{*}(=1)$ the maximum dimensionless mole fraction of material $A, C_{A, x}^{*}$ the dimensionless mole fraction distribution of material $A$ at the exit, $x$ the local coordinate at exit, and $L$ the width of channel exit. When the value of $\varepsilon_{\text {mixing }}$ approaches to 1 , the mixing efficiency is getting better, whereas the mixing efficiency approaches to 0 , the mixing efficiency is getting worse.

\section{Results and Discussions}

\subsection{The simple Y-type micromixer}

First, numerical computations are performed for a micromixer with simple geometry, i.e., a Y-type micromixer without adding a nozzle and feedback side-channels. This case is denoted as " 0 cyc- 0 nozzle". The width of inlet channels $\left(D_{1}\right)$ and main channel $\left(D_{2}\right)$ are $500 \mu \mathrm{m}$ and $400 \mu \mathrm{m}$, respectively. The inlet velocities $\left(V_{0}\right)$ vary from

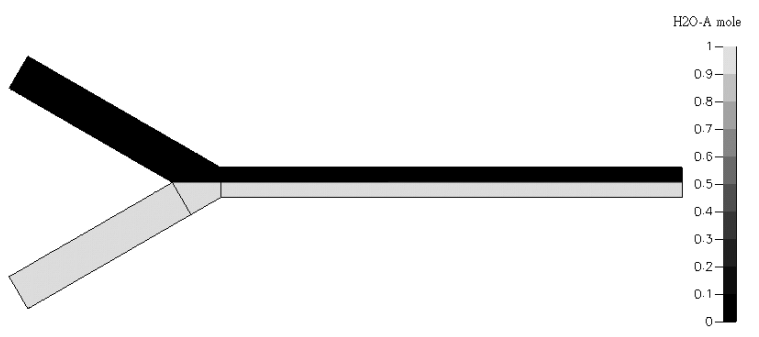

Fig. 2 The mixing effect of " 0 cyc- 0 nozzle" case for $R e=30$

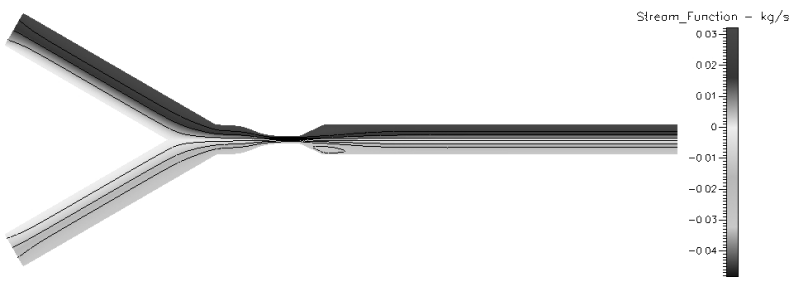

(a)

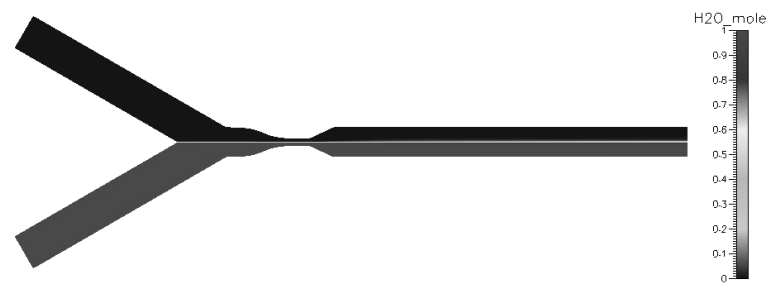

(b)

Fig. 3 Simulation results for " 0 cyc-1 nozzle" case at $R e=30$. (a) Streamlines and (b) Concentration distributions

$0.01 \mathrm{~m} / \mathrm{s}$ to $0.06 \mathrm{~m} / \mathrm{s}$ with an increment of $0.005 \mathrm{~m} / \mathrm{s}$, and therefore, the corresponding Reynolds number is ranged from $R e=5$ to $R e=30$ with an increment of 2.5. With this simple geometry, all the numerical computations show that no flow oscillations are found in the main channel. The mole fraction distribution at the exit of main channel shows a clear-cut situation and there is only few mixing in the middle of main channel. Figure 2 shows the computational result of a simple Y-type micromixer for $R e=30$. It is found that the mixing effect is poor and is worse for higher Reynolds number.

\subsection{Addition of convergent nozzle}

It is considered that a modification of original Y-type micromixer by adding a convergent nozzle at the top of main channel and it is denoted as " 0 cyc- 1 nozzle" case in this study. The geometry design of the convergent nozzle can be referred to Ref.(9). Figure 3 represents the computed concentration field for the case of $R e=30$. It shows that the mixing effect is still limited. The pressure drops and mixing efficiencies for both " 0 cyc-0 nozzle" and " 0 cyc- 1 nozzle" cases are shown in Fig. 4 (a) and (b), respectively. It is seen that the mixing effect can only be slightly increased with the addition of a convergent nozzle, but the pressure drop is largely increased for " 0 cyc- 1 nozzle" case. 


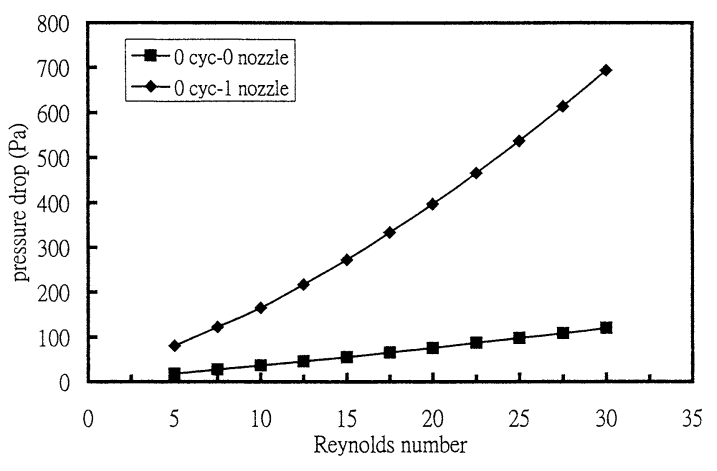

(a)

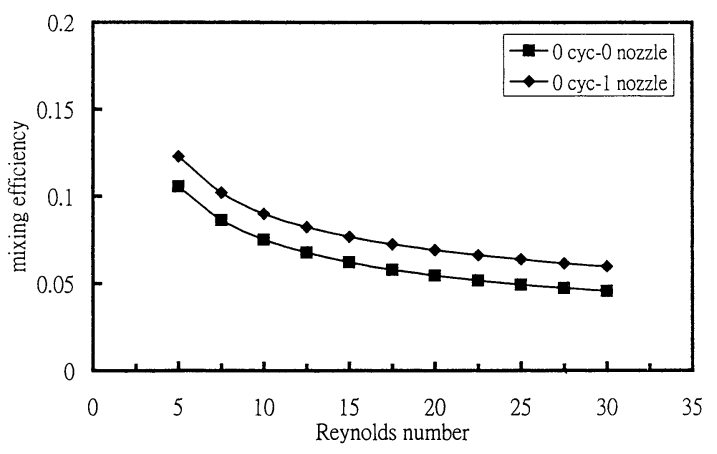

(b)

Fig. 4 Comparison of (a) pressure drop and (b) mixing efficiency for " 0 cyc- 0 nozzle" case and " 0 cyc- 1 nozzle" case

\subsection{Symmetric arrangement of side channels}

In order to improve the mixing effect, a new geometry design is proposed in this study such that the fluid flow in the main channel can be effectively disturbed. First, the feedback side channels are symmetrically added to both sides of the main channel. The schematic diagram, as depicted in Fig. 1, is denoted as " 1 cyc-sym" case. The maximum width of the side-channel is $200 \mu \mathrm{m}$. The exit of the side-channel, which is orthogonal to the main channel, is designed to be a convergent nozzle so as to accelerate the fluid and then, the flow in main channel can be disturbed as expected. Numerical computations are performed with this geometry for $R e=5$ to $R e=30$. The mole-fraction distributions at the exit for various Reynolds number are shown in Fig. 5. It is found that, for $R e \leq 20$, the mixing effect is getting worse with an increasing Reynolds number, but it is getting better with a larger Reynolds number for $R e>20$. It is noted that there is a sudden increase of mixing efficiency for Reynolds number larger than 20 .

The variation of pressure drop and mixing efficiency with respect to Reynolds number for " 0 cyc-1 nozzle" and "1 cyc-sym" cases are shown in Fig. 6 (a) and (b), respectively. It can be seen that the pressure drop for " 1 cycsym" case is only slightly higher than that of " 0 cyc- 1 nozzle" case, but the mixing efficiency for " 1 cyc-sym" case has a sudden increase for Reynolds number being larger than 20 . The mixing efficiency of " 0 cyc -1 nozzle" case

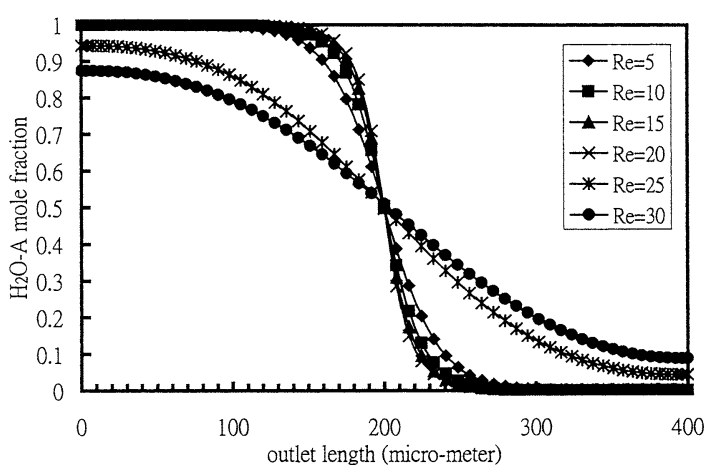

Fig. 5 The mole-fraction distribution at the main-channel exit with varying Reynolds numbers for one cycle of symmetrical side channel

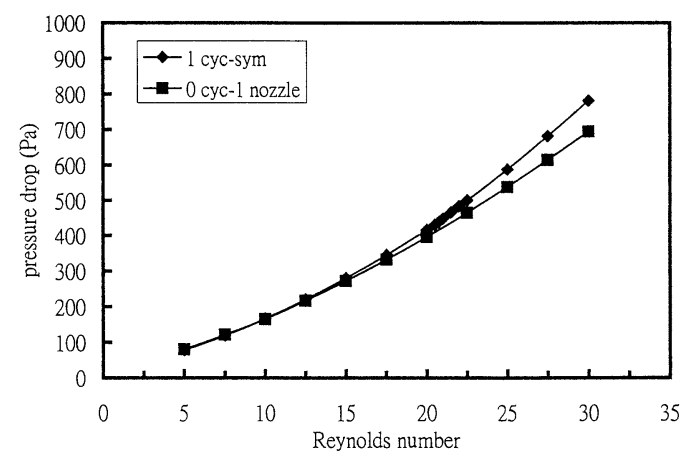

(a)

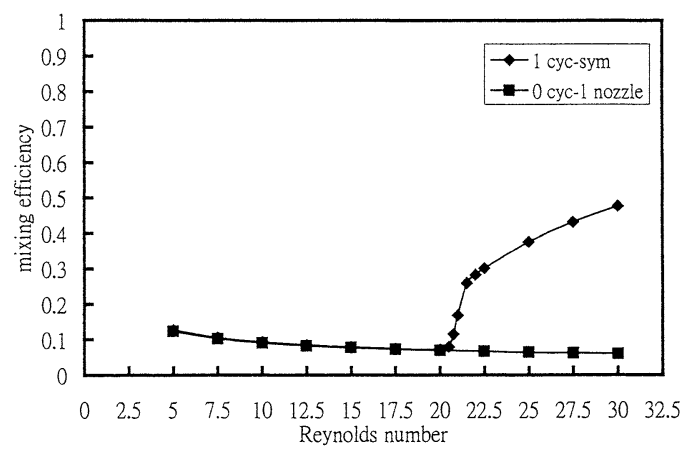

(b)

Fig. 6 Comparisons of (a) the pressure drop and (b) the mixing efficiency between " 0 cyc- 1 nozzle" case and " 1 cycsym" case

for $R e=30$ is 0.0597 , whilst it is eight times for " 1 cycsym" case, i.e., the mixing efficiency is 0.4773 . To understand why the mixing efficiency is enhanced with the addition of feedback side channels, the plots of streamline and concentration distribution for " 1 cyc-sym" case at $R e=30$ are provided in Fig. 7. Comparing with " 0 cyc -1 nozzle" case as shown in Fig. 3, it can be seen that an apparent vortex is formed for the "1cyc-sym" case, and therefore, the mixing effect at the main-channel exit is getting better. The reason for the improvement of mixing effect is that the velocity of fluid flowing out from the side channels becomes higher with an increasing Reynolds number, and 


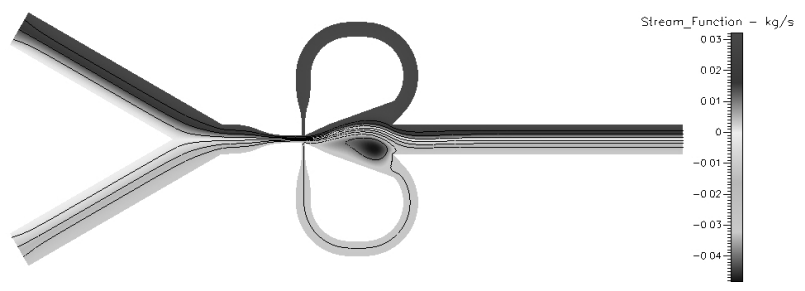

(a)

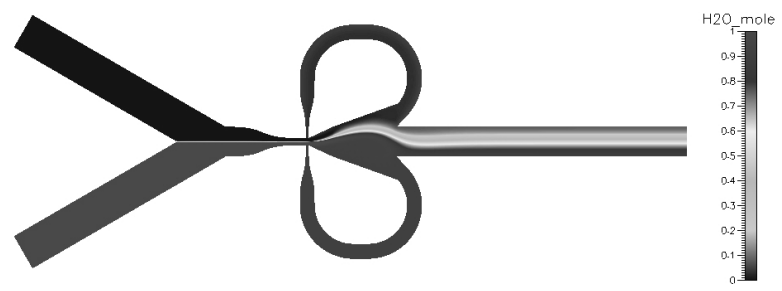

(b)

Fig. 7 Simulation results for "1 cyc-sym" case at $R e=30$. (a) Streamlines and (b) Concentration distributions

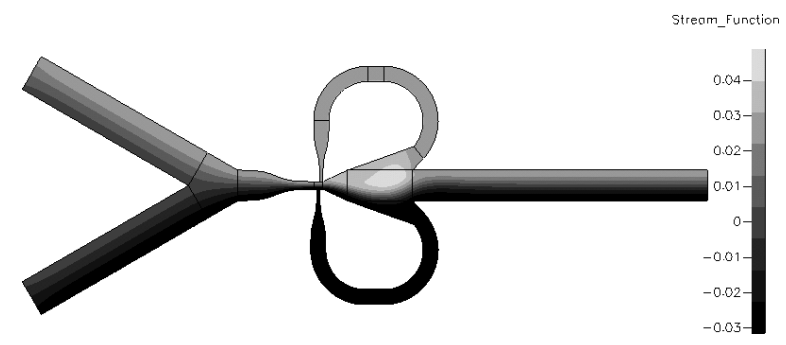

(a)

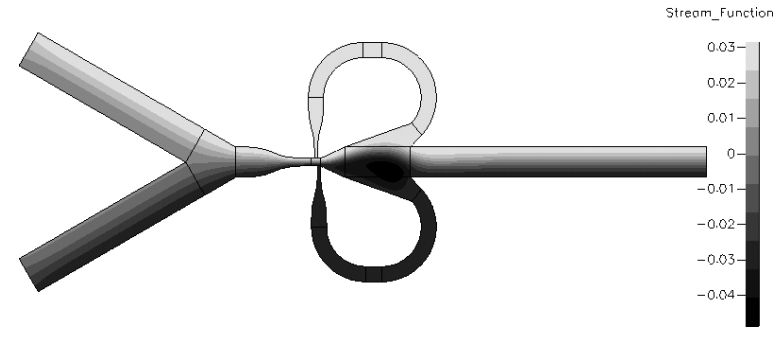

(b)

Fig. 8 The position of vortex for (a) "1cyc-L40" case and (b) "1cyc-R-40" case

therefore, the main stream can be effectively disturbed by the fluids flowing out from the side channels.

\subsection{Asymmetric arrangement of side channels}

To control the position of the vortex formed in the mixing chamber and their influences on the mixing effect, the exits of side channels can be asymmetrically arranged. In this study, "L-40" and "L-80" denote the cases that the exit of left side-channel is raised a distance of $40 \mu \mathrm{m}$ and $80 \mu \mathrm{m}$ higher than the exit position of right side-channel, respectively. Similarly, "R-40" and "R-80" mean that the exits of right side-channel are $40 \mu \mathrm{m}$ and $80 \mu \mathrm{m}$ higher than that of left side-channel, respectively. Numerical simulations show that the pressure drop and mixing efficiency for "L-40" case and "R-40" case are of the same values, but the flow structures are different, as shown in Fig. 8 (a)

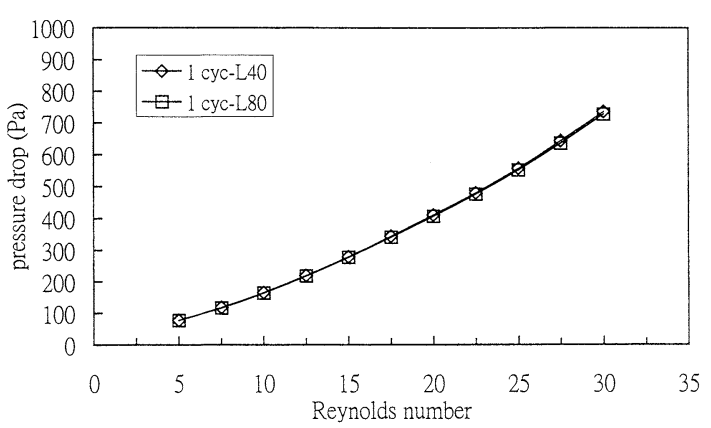

(a)

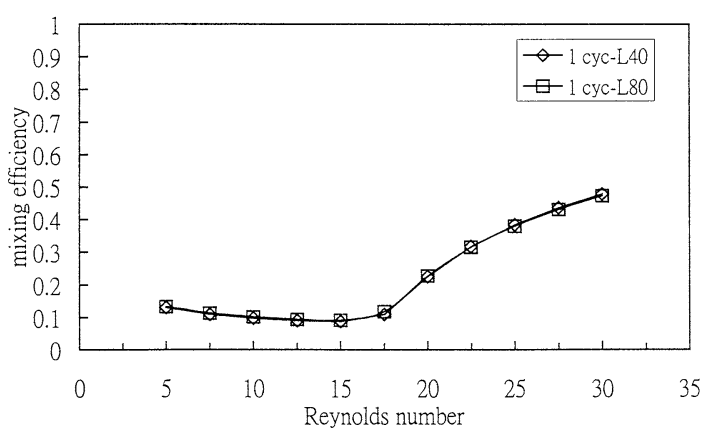

(b)

Fig. 9 Comparisons of (a) the pressure drop and (b) the mixing efficiency between "1cyc-L40" case and "1cyc-L80" case

and (b). Figure 8 (a) shows the vortex is on the right for "L-40" case and it is on the left for "R-40" case, as shown in Fig. 8(b). Therefore the position where the vortex is formed can be adjusted through the asymmetric arrangement of side-channel exits. This provides a design concept for the flow oscillation mechanism in a micromixer, i.e. the flow will be bended due to the multiple side-channel arrangements. The influence of the shifted distance between the exits of left and right side-channels on the mixing effect is not significant. The pressure drop and mixing efficiency for cases "L-40" and "L-80" are shown in Fig. 9 (a) and (b), respectively, for $R e=30$. It is found that the two curves in each of these figures approximately coincide. The pressure drop for cases "L-40" and "L-80" are 735.702 $\mathrm{Pa}$ and $727.521 \mathrm{~Pa}$, respectively. The difference is decreased only of $8.181 \mathrm{~Pa}$ (i.e., 1.1\%). The mixing efficiency is 0.4789 for the "L-40" case and 0.4736 for "L-80"case. It is only a small difference of 0.0053 (i.e., $1.1 \%)$.

Figure 10 (a) and (b) show the comparison of pressure drop and mixing efficiency, respectively, among various geometries. The total length of main channel is kept fixed for all cases. Results show that the pressure drops are approximately of equal values for cases with or without side channels arranged symmetrically or asymmetrically. The pressure drop of the simple Y-shape case is of the lowest value. The diagram of mixing efficiency shows an interesting result that the asymmetrical arrangement of 


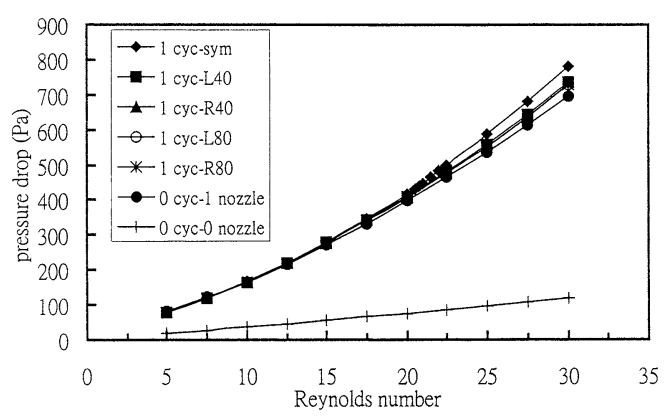

(a)

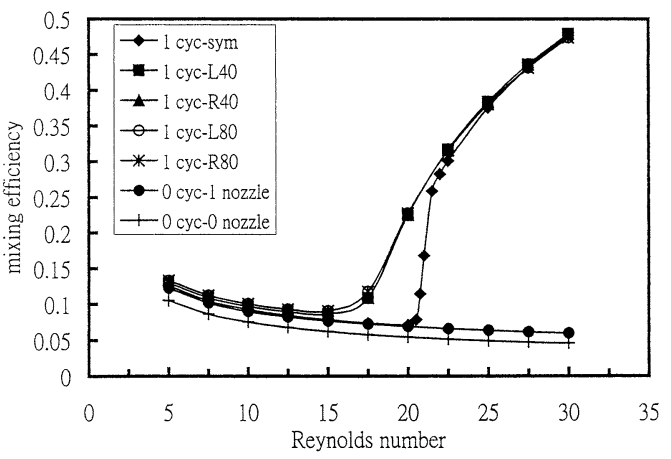

(b)

Fig. 10 Comparison of (a) pressure drop and (b) mixing efficiency for various design geometries under the fixed length of one side-channel cycle

Table 1 Comparison of mixing efficiency of two-side-channel case for various Reynolds number

\begin{tabular}{|c|c|c|c|c|c|c|c|}
\hline & $\mathrm{Re}=15.0$ & $\mathrm{Re}=17.5$ & $\mathrm{Re}=20.0$ & $\mathrm{Re}=22.5$ & $\mathrm{Re}=25.0$ & $\mathrm{Re}=27.5$ & $\mathrm{Re}=30.0$ \\
\hline 0 cycle & 0.0999 & 0.0954 & 0.0917 & 0.0886 & 0.0857 & 0.0832 & 0.0810 \\
\hline nozzle & & & & & & & \\
\hline 2sym & 0.1039 & 0.0981 & 0.0956 & 0.4647 & 0.5745 & 0.6484 & 0.7025 \\
\hline L40L40 & 0.1169 & 0.1530 & 0.3390 & 0.4875 & 0.5830 & 0.6505 & 0.7010 \\
\hline R40R40 & 0.1169 & 0.1530 & 0.3390 & 0.4875 & 0.5830 & 0.6505 & 0.7010 \\
\hline L80L80 & 0.1232 & 0.1666 & 0.3438 & 0.4847 & 0.5778 & 0.6442 & 0.6945 \\
\hline R80R80 & 0.1232 & 0.1666 & 0.3438 & 0.4847 & 0.5778 & 0.6442 & 0.6945 \\
\hline L40R40 & 0.1169 & 0.1534 & 0.3388 & 0.4874 & 0.5842 & 0.6526 & 0.7037 \\
\hline R40L40 & 0.1169 & 0.1534 & 0.3388 & 0.4874 & 0.5842 & 0.6526 & 0.7037 \\
\hline L80R80 & 0.1232 & 0.1670 & 0.3435 & 0.4848 & 0.5793 & 0.6468 & 0.6976 \\
\hline R80L80 & 0.1232 & 0.1670 & 0.3435 & 0.4848 & 0.5793 & 0.6468 & 0.6976 \\
\hline
\end{tabular}

side channels can improve the mixing effect at a smaller Reynolds number. It means that the pumping power can be smaller to achieve a better mixing for the asymmetrical arrangement.

\subsection{Effects of number of cycles of side channels}

It has been shown that the mixing effect can be improved by the addition of side channels. The mixing efficiency can be increased further by the addition of more cycles of feedback side-channels. Tables 1-3 present the comparison of mixing efficiency for two-, four, and six cycles of side channels. The length of design part will be increased as the number of added side-channels increases,
Table 2 Comparison of mixing efficiency of four-side-channel case for various Reynolds number

\begin{tabular}{|c|c|c|c|c|c|c|c|}
\hline & $\mathrm{Re}=15.0$ & $\mathrm{Re}=17.5$ & $\mathrm{Re}=20.0$ & $\mathrm{Re}=22.5$ & $\mathrm{Re}=25.0$ & $\mathrm{Re}=27.5$ & $\mathrm{Re}=30.0$ \\
\hline $\begin{array}{c}\text { 0 cycle } \\
\text { nozzle }\end{array}$ & 0.1421 & 0.1380 & 0.1349 & 0.1321 & 0.1297 & 0.1274 & 0.1247 \\
\hline 4sym & 0.1527 & 0.1455 & 0.1435 & 0.6907 & 0.8056 & 0.8650 & 0.9027 \\
\hline $\begin{array}{c}\mathrm{L} 40 \mathrm{~L} 40 \\
\mathrm{~L} 40 \mathrm{~L} 40\end{array}$ & 0.1730 & 0.2310 & 0.5253 & 0.7152 & 0.8106 & 0.8662 & 0.9017 \\
\hline $\begin{array}{l}\mathrm{R} 40 \mathrm{R} 40 \\
\mathrm{R} 40 \mathrm{R} 40\end{array}$ & 0.1730 & 0.2310 & 0.5253 & 0.7152 & 0.8106 & 0.8662 & 0.9017 \\
\hline $\begin{array}{c}\mathrm{L} 80 \mathrm{~L} 80 \\
\mathrm{~L} 80 \mathrm{~L} 80\end{array}$ & 0.1828 & 0.2518 & 0.5315 & 0.7114 & 0.8053 & 0.8610 & 0.8971 \\
\hline $\begin{array}{l}\mathrm{R} 80 \mathrm{R} 80 \\
\mathrm{R} 80 \mathrm{R} 80\end{array}$ & 0.1828 & 0.2518 & 0.5315 & 0.7114 & 0.8053 & 0.8610 & 0.8971 \\
\hline $\begin{array}{c}\mathrm{L} 40 \mathrm{R} 40 \\
\mathrm{~L} 40 \mathrm{R} 40\end{array}$ & 0.1730 & 0.2314 & 0.5249 & 0.7170 & 0.8142 & 0.8702 & 0.9054 \\
\hline $\begin{array}{l}\mathrm{R} 40 \mathrm{~L} 40 \\
\mathrm{R} 40 \mathrm{~L} 40\end{array}$ & 0.1730 & 0.2314 & 0.5249 & 0.7170 & 0.8142 & 0.8702 & 0.9054 \\
\hline $\begin{array}{l}\mathrm{L} 80 \mathrm{R} 80 \\
\mathrm{~L} 80 \mathrm{R} 80\end{array}$ & 0.1827 & 0.2522 & 0.5311 & 0.7139 & 0.8097 & 0.8659 & 0.9016 \\
\hline $\begin{array}{l}\mathrm{R} 80 \mathrm{~L} 80 \\
\mathrm{R} 80 \mathrm{~L} 80\end{array}$ & 0.1827 & 0.2522 & 0.5311 & 0.7139 & 0.8097 & 0.8659 & 0.9016 \\
\hline
\end{tabular}

Table 3 Comparison of mixing efficiency of six-side-channel case for various Reynolds number

\begin{tabular}{|c|c|c|c|c|c|c|c|}
\hline & $\mathrm{Re}=15.0$ & $\operatorname{Re}=17.5$ & $\mathrm{Re}=20.0$ & $\operatorname{Re}=22.5$ & $\operatorname{Re}=25.0$ & $\mathrm{Re}=27.5$ & $\mathrm{Re}=30.0$ \\
\hline $\begin{array}{l}0 \text { cycle } \\
6 \text { nozzle }\end{array}$ & 0.1831 & 0.1802 & 0.1782 & 0.1764 & 0.1746 & 0.1726 & 0.1701 \\
\hline 6sym & 0.2016 & 0.1933 & 0.1897 & 0.8221 & 0.9170 & 0.9481 & 0.9687 \\
\hline $\mathrm{L} 40 \times 6$ & 0.2282 & 0.3049 & 0.6614 & 0.8419 & 0.9139 & 0.9487 & 0.9677 \\
\hline $\mathrm{R} 40 \times 6$ & 0.2282 & 0.3049 & 0.6614 & 0.8419 & 0.9139 & 0.9487 & 0.9677 \\
\hline L80 $\times 6$ & 0.2410 & 0.3314 & 0.6675 & 0.8385 & 0.9101 & 0.9456 & 0.9653 \\
\hline $\mathrm{R} 80 \times 6$ & 0.2410 & 0.3314 & 0.6675 & 0.8385 & 0.9101 & 0.9456 & 0.9653 \\
\hline $\begin{array}{c}(\mathrm{L} 40 \mathrm{R} 40) \\
\times 3\end{array}$ & 0.2281 & 0.3052 & 0.6610 & 0.8441 & 0.9170 & 0.9515 & 0.9698 \\
\hline $\begin{array}{c}(\mathrm{R} 40 \mathrm{~L} 40) \\
\times 3\end{array}$ & 0.2281 & 0.3052 & 0.6610 & 0.8441 & 0.9170 & 0.9515 & 0.9698 \\
\hline $\begin{array}{c}\text { (L80R80) } \\
\times 3\end{array}$ & 0.2409 & 0.3318 & 0.6675 & 0.8415 & 0.9140 & 0.9491 & 0.9680 \\
\hline $\begin{array}{c}(\mathrm{R} 80 \mathrm{~L} 80) \\
\times 3\end{array}$ & 0.2409 & 0.3318 & 0.6675 & 0.8415 & 0.9140 & 0.9491 & 0.9680 \\
\hline
\end{tabular}

and the length of L2, see Fig. 1 (a), is kept constant for all design cases. It is shown that the more side channels are added, the higher the mixing efficiency will be. It can be found that, with six cycles of side channels are added, the mixing efficiency can be as high as 0.9698 . Figure 11 (a) and (b) display the effect of number of cycles on the pressure drop and mixing efficiency for the symmetrical arrangement of side channels. It is clearly shown that the pressure drop is increased monotonically 


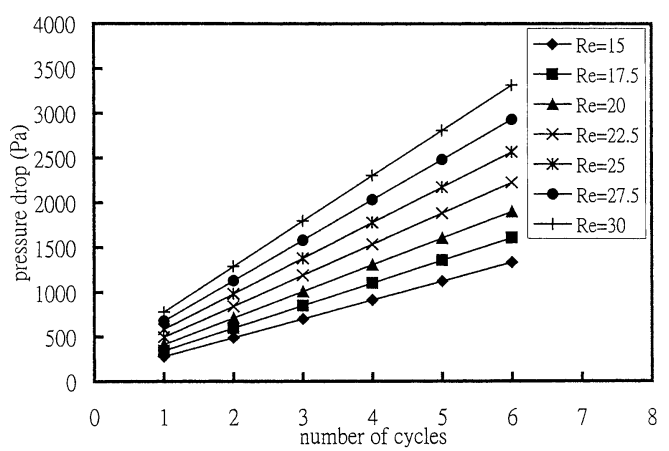

(a)

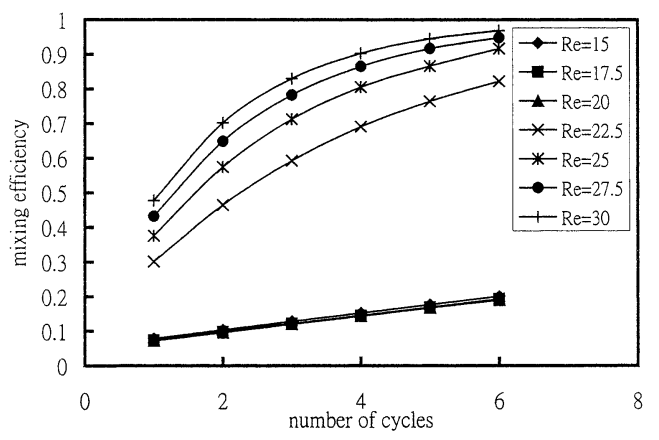

(b)

Fig. 11 Effect of number of cycles of feedback side-channels on (a) pressure drop and (b) mixing efficiency for symmetrical case

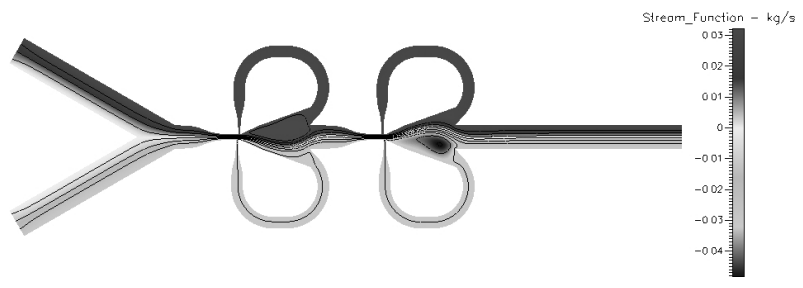

(a) "2 cyc-L40R40" case

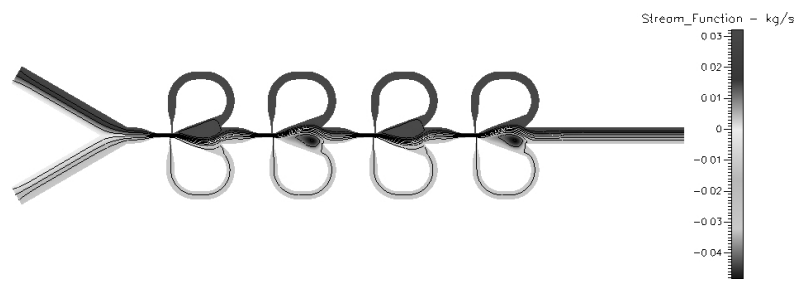

(b) "4 cyc-L40R40L40R40" case

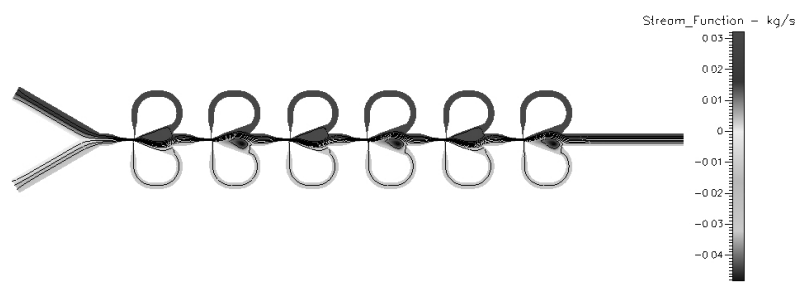

(c) "6 cyc-L40R40L40R40L40R40" case

Fig. 12 The streamlines for cases with 2, 4, and 6 side-channels at $\operatorname{Re}=30$

with the increasing number of cycles of side channels and Reynolds number. The streamlines and concentration dis-

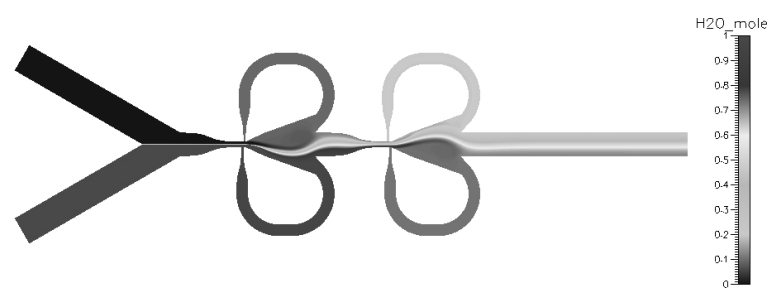

(a) "2 cyc-L40R40" case

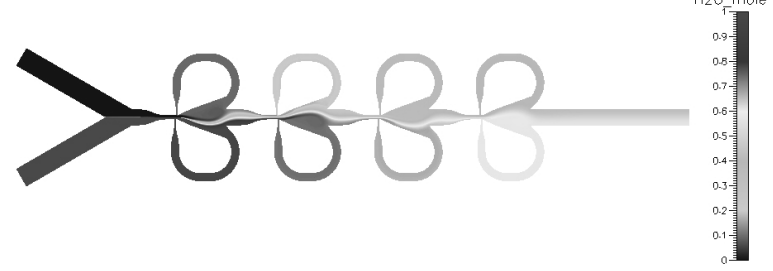

(b) “4 cyc-L40R40L40R40” case

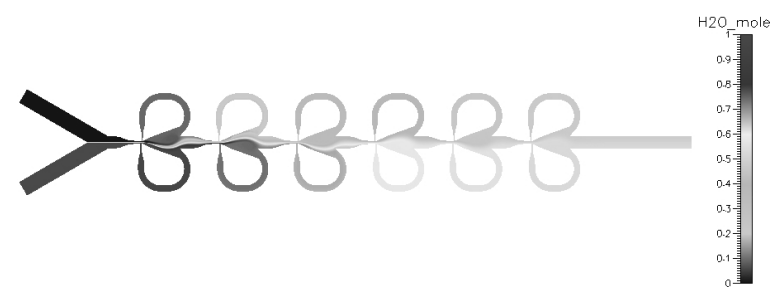

(c) "6 cyc-L40R40L40R40L40R40" case

Fig. 13 Concentration distributions for cases with 2, 4, and 6 side-channels at $R e=30$

tributions for cases with 2,4 , and 6 side-channels are presented, in Figs. 12 and 13, to show that the mixing efficiency increases with an increasing number of added sidechannels. The detailed physical phenomena need a further study.

\section{Conclusions}

A new type of micromixer is proposed and numerical simulations of flow fields in the new micromixer are performed. Major findings of this work can be stated as follows:

(1) A simple Y-type micromixer, with or without a convergent nozzle, can only have molecular diffusion in the middle of main channel. The mixing between different fluids is poor.

(2) The addition of feedback side channels does improve the mixing effects. The mixing efficiency is increased with the increasing Reynolds number and there is a mixing enhancement for Reynolds number between 20 and 22.5 .

( 3 ) The pressure drop and mixing efficiency for symmetrical and asymmetrical arrangements of side channels are approximately equal. The flow structures, i.e., the position of vortex, for higher left side-channel and higher right side-channel designs are different.

(4) As the number of cycles of side channels is increased, the mixing efficiency increases and approaches to unity for the effective Reynolds number, i.e., the Reynolds 
number is greater than 20 .

\section{Acknowledgment}

The first author (CIH) would like to thank the reviewers for valuable comments and Mr. W.S. Lin for preparation some of the figures of the revised manuscript. Valuable suggestions from Prof. S.T. Choi at Dept. of IAA, NCKU are also appreciated.

\section{References}

( 1 ) Manz, A., Graber, N. and Widmer, H.M., Miniaturized Total Chemical Analysis Systems: A Novel Concept for Chemical Sensing, Sensors Actuators, Vol.B1 (1990), pp.244-248.

( 2 ) Yang, Z., Goto, H., Matsumoto, M. and Yada, T., Micromixer Incorporated with Piezoelectrially Driven Valveless Micropump, Micro Total Analysis Systems'98, (1998), pp.177-180.

( 3 ) Yang, Z., Goto, H., Matsumoto, M. and Maeda, R., Ultrasonic Micromixer for Microfluidic Systems, Electrophoresis, Vol.21 (2000), pp.116-119.
( 4 ) Miyake, R., Lammerink, T.S.J., Elwenspoek, M. and Fluitman, J.H.J., Micro Mixer with Fast Diffusion, IEEE MEMS'93, (1993), pp.248-253.

( 5 ) Knight, J.B., Vishwanath, A., Brody, J.P. and Austin, R.H., Hydrodynamics Focusing on a Silicon Chip: Mixing Nanoliters in Microseconds, Physical Review Letters, Vol.80 (1998), pp.3863-3866.

( 6 ) Schwesinger, N., Frank, T. and Wurmus, H., A Modular Microfluid System with an Integrated Micromixer, J. Micromech. Microeng., Vol.6 (1996), pp.99-102.

( 7 ) Veenstra, T.T., Lammerink, T.S.J., Elwenspoek, M.C. and van den Berg, A., Characterization Method for a New Diffusion Mixer Applicable in Micro Flow Injection Analysis Systems, J. Micromech. Microeng., Vol.9 (1999), pp.199-202.

( 8 ) CFD-ACE(U) User's Manual, Version 6.6, CFD Research Corporation, (1998).

( 9 ) Wang, K.C., Design and Flow Simulation of a New Type Micromixer, Master Thesis, (in Chinese), Department of Mechanical Engineering, National ChengKung University, Tainan, Taiwan, (2002). 